\title{
What's new for the clinician? \\ - Excerpts from and summaries of recently published papers
}

\section{Comparison of the effect of therapeutic elastic bandage, submucosal dexamethasone, or dexketoprofen trometa- mol on inflammatory symptoms and quality of life following third molar surgery}

A Erdil, N Akbulut, A Altan, MS Demirsoy. Comparison of the effect of therapeutic elastic bandage, submucosal dexamethasone, or dexketoprofen trometamol on inflammatory symptoms and quality of life following third molar surgery: A randomized clinical trial. Clinical Oral Investigations. 2021; 25: 1849-57

\section{INTRODUCTION}

Extraction of third molars is one of the most common procedures performed by oral surgeons. Generally, these surgeries do not encounter difficulties but at times can result in complications. Postoperative complications may include swelling, pain, trismus, prolonged bleeding, dry socket, infection and sensory alterations of the inferior alveolar nerve or lingual nerve. For the swelling, the most common therapies include corticosteroids, non-steroid anti-inflammatory drugs (NSAIDs), or a combination of these agents.

Elastic bandage application (Kinesio taping) is a technique that originates from sports sciences. Studies have reported its beneficial effects on swelling and trismus symptoms after oral and maxillofacial surgeries. ${ }^{1}$ It has been claimed that elastic tapes reduce postoperative swelling by creating space between the dermis and fascia layers and thus increasing lymphatic and blood flow. ${ }^{1}$ Their alleviating effect on postoperative pain is due to dermal stimulation. ${ }^{1}$ Erdil and colleagues $(2021)^{1}$ reported on a trail that sought to compare the effectiveness of submucosal dexamethasone injection, a therapeutic elastic bandage (Kinesio tape), and an NSAID (dexketoprofen trometamol) on postoperative swelling, trismus, pain following third molar surgery and alterations in the quality of life in the first postoperative week.

Veerasamy Yengopal: $B C h D, B S c H o n s, M C h D, P h D$, Community Dentistry Department, School of Oral Health Sciences, University of Witwatersrand, Medical School, no. 7 York Road, Parktown 2193, South Africa.

ORCID Number: 0000-0003-4284-3367

Email: veerasamy.yengopal@wits.ac.za

\section{MATERIALS AND METHODS}

The inclusion criteria for this randomized clinical trial were healthy adult patients, age between 18 and 65 years who required surgical removal of impacted third molars.

Patients who were outside the age range, were smokers, had been diagnosed with chronic systemic and psychiatric diseases, had received antibiotic therapy or analgesic therapy for related impacted teeth in the last 30 days, or presented with active complaints on the surgery day and patients with a history of allergy to any of the medications or devices utilized in the study and whose total surgery time exceeded $45 \mathrm{~min}$ were excluded.

The participants were randomized into three groups:

- Corticosteroid group (CS): Preoperative submucosal $8 \mathrm{mg} / 2 \mathrm{ml}$ dexamethasone injection

- Therapeutic elastic bandage application group (KT): Postoperative Kinesio tape application

- Non-steroid anti-inflammatory drug group (DT): Postoperative $25 \mathrm{mg}$ dexketoprofen trometamol prescription.

Inferior alveolar, lingual, and buccal nerve blockades were obtained with $2 \mathrm{ml}$ of a local anaesthetic containing 40 $\mathrm{mg} / \mathrm{ml}$ articaine and $0.005 \mathrm{mg} / \mathrm{ml}$ epinephrine (Maxicaine) in all groups. For the patients in the Corticosteroid group (CS) group, $2 \mathrm{ml}$ of a solution containing $8 \mathrm{mg}$ dexamethasone 21-phosphate was injected at the depth of the buccal sulcus near the operation site after local anaesthesia. One mandibular third molar was extracted from each participant, and all surgeries were performed by the same experienced practitioner. 
In the Kinesio tape (KT) group, after completion of the surgeries, the 2-inch-wide tapes were measured as the distance between the tragus-commissure line and clavicle, individually. The arranged tapes were cut into five equal pieces and applied to the operated sides to cover the submental, mandibular, submandibular, preauricular, parotid, and superficial cervical lymph node regions. The tapes were removed on the second postoperative day.

All the patients were given the same postoperative recommendations, but none of them applied ice packs. Amoxicillin + clavulanic acid $625 \mathrm{mg}$ (twice a day for 5 days) and chlorhexidine gluconate + benzydamine hydrochloride mouthwash (every $8 \mathrm{~h}$ for 7 days) were prescribed for all groups to reduce the effect of infection on the postoperative course as much as possible.

Paracetamol $500 \mathrm{mg}$ (every $8 \mathrm{~h}$ for 7 days) was prescribed for the patients in the CS and KT groups. Dexketoprofen trometamol $25 \mathrm{mg}$ (every $8 \mathrm{~h}$ for 7 days) was prescribed for patients in the DT group, and paracetamol $500 \mathrm{mg}$ was prescribed as a rescue analgesic. The participants were advised not to exceed 6 tablets per day to avoid acute liver failure.

The study variables were classified as demographic, anatomic, and outcome variables. The demographic variables were age, and gender, the anatomic variable was the surgical difficulty. The primary outcomes were pain intensity, trismus, and oedema. The healing rates of surgical sites and the quality of life (QoL) scores were considered as secondary outcomes. In all groups, baseline characteristics and demographic parameters were observed preoperatively on surgery day (TO), and inflammatory symptoms and healing rates of operated sites were evaluated on the postoperative second (T1) and seventh (T2) days. The total operation time was recorded individually to specify the surgical difficulty, and patients whose operation time exceeded 45 min were excluded.

Baseline facial dimensions were measured with a tape measure between the reference points of the traguslateral commissure and lateral canthus-angulus on the operated sites. Oedema was indicated by an increase in the baseline mean distances.

Trismus was observed as a decrease in preoperatively measured maximal mouth opening, which was measured as the distance between the upper and lower right or left central incisors.

Pain intensity was evaluated subjectively using a Numerical Rating Scale (NRS) on which "0" means no pain, "5" means moderate pain, and "10" means worst imaginable pain. Additionally, analgesic consumption was individually recorded as tablets per day for the T0-T1 and T1-T2 intervals, and the total postoperative period.

Quality of life (QoL) was measured at T0, T1, and T2 in all groups using the Oral Health Impaction Profile-14 $(\mathrm{OHIP}-14)$ scale where each item is scored as 0 , never; 1, hardly ever; 2, occasionally; 3 , fairly often; and 4, very often. Total scores vary between 0 (absence of problems) and 56 (experiencing the mentioned problems very often).
For healing, the extraction sites were examined in terms of tissue colour, response to palpation, existing granulation tissue, and condition of the incision margin and scored as 1, very poor; 2, poor; 3, good; 4, very good; and 5, excellent. From this, a healing score was determined.

The postoperative 1-week period from the viewpoint of the participants was evaluated with the Postoperative Symptom Severity Scale (PoSSe) on T2. PoSSe contains seven subscales and 15 questions that were devised from common clinical questions to assess symptoms of the patients with third molar extractions. The obtainable minimum score is $0 \%$ (least severe response), and the maximum score is $100 \%$ (most severe response).

\section{RESULTS}

The study population consisted of 57 patients. Of this, five patients (two for exceeded surgery time, three for loss to follow-up) were excluded. The remaining patients completed the study protocol and the requested questionnaires. Data obtained from 52 patients were analysed; 36 were female, 16 were male, and the mean age was 24.9 years. After exclusion, the groups consisted of 17 patients in the dexketoprofen trometamol (DT) group, 16 patients in the Corticosteroid group (CS) group, and 19 patients in the Kinesio tape (KT) group. No significant difference was found between the three groups regarding baseline variables. Neither participant experienced complications such as alveolar osteitis, infection, dehiscence, sensorial disturbance, or any other postoperative complication.

The preoperative mean maximal mouth opening was 42 $\mathrm{mm}$. In all groups, a reduction in mouth opening was detected on $\mathrm{T} 1$, with a statistically significant difference between the DT group and the other groups $(p=0.002)$. The mean restriction in mouth opening was $21 \mathrm{~mm}$ in the DT group, $12 \mathrm{~mm}$ in the CS group $(p=0.003)$, and $16 \mathrm{~mm}$ in the KT group $(\mathrm{p}=0.014)$.

The most severe oedema accumulation was observed in the DT group on T1, and a statistically significant difference was found with the other groups $(p=0.014)$. However, on T2, the difference between groups was statistically insignificant $(p=0.112)$. Compared with baseline levels on T2, swelling persisted mildly in the DT group and approached the baseline values in both the CS and the KT groups.

There was a statistically significant difference in total mean pain rating (NRS) scores between the KT group and the other groups $(p=0.001)$. On $T 1$, there was no statistically significant difference between any of the groups. On T2, there was a significant difference between the KT group and the other groups $(p=0.010)$. Regarding analgesic consumption, at all-time intervals, the DT group consumed significantly more tablets than the other groups.

The clinically assessed healing rates significantly differed between groups on $\mathrm{T} 1$ and $\mathrm{T} 2(\mathrm{p}=0.015, \mathrm{p}=0.047$, respectively). On T1, the lowest scores were obtained in the DT group; however, on T2, the CS group had the lowest scores. 
According to the subjective assessment of QoL in the postoperative period, total mean OHIP-14 scores did not differ between groups on T0 $(p=0.497), T 1 \quad(p=0.217)$, and T2 $(p=0.969)$. Nevertheless, the mean total scores in all three groups decreased on T2 compared with T0 and T1. On T2, there was a significant difference between the groups in terms of psychological discomfort $(p=0.027)$; the dexketoprofen trometamol (DT) and Corticosteroid group (CS) groups were similar to each other $(p>0.999)$. In contrast, the Kinesio tape (KT) group significantly differed from the DT group $(p=0.039)$. However, the total mean PoSSe and eating subscale scores differed significantly between groups $(p=0.016, p=0.032)$. The participants involved in the DT group reported the highest scores, whereas the participants in the KT and CS groups exhibited similar results.

\section{CONCLUSIONS}

This trail found that Kinesio taping could provide results in terms of trismus, oedema, and QoL that were similar to those of a corticosteroid with long-acting and high glucocorticoid potency. However, Kinesio taping was less effective than dexamethasone for postoperative pain.

\section{Implications of practice}

In cases where NSAIDs (anti-inflammatory medication) and corticosteroids are contraindicated, or additional anti-inflammatory measures are required, the therapeutic elastic bandage application appears promising and should be considered as a supportive care modality. The combination of Kinesio taping with other pain-relieving methods should provide benefits similar to that of using corticosteroids in patients who have undergone third molar surgery.

\section{Reference}

1. Erdil A, Akbulut N, Altan A, Demirsoy MS. Comparison of the effect of therapeutic elastic bandage, submucosal dexamethasone, or dexketoprofen trometamol on inflammatory symptoms and quality of life following third molar surgery: A randomized clinical trial. Clinical Oral Investigations. 2021; 25: $1849-57$.

\section{Evaluation of the effect of probiotic lozenges in the treatment of recurrent aphthous stomatitis: A RCT}

$\mathrm{RL}$ Aggour, SH Mahmoud, A Abdelwhab. Evaluation of the effect of probiotic lozenges in the treatment of recurrent aphthous stomatitis: a randomized, controlled clinical trial. Clinical Oral Investigations. 2021; 25: 2151-8.

\section{INTRODUCTION}

Recurrent aphthous stomatitis (RAS) is the most common painful oral mucosal disease. It affects nonkeratinized mucosa in men and women of all ages, races, and geographic regions. Minor RAS is the most common form, which accounts for approximately 70 to $87 \%$ of the population with RAS and usually has 1 to 5 ulcers at one episode, with a size of less than $1 \mathrm{~cm}$ in diameter. ${ }^{1}$ For RAS patients, the ulcer pain associated with each episode may severely interfere with eating, speaking, and swallowing. The aetiology of RAS remains unknown and thus all current systemic or topical treatment methods are to relieve symptoms and accelerate healing. Most systemic medications, although effective, have side effects that limit their general use. Therefore, topical agents remain the first choice for the treatment of RAS, due to their effectiveness and safety.

The application of host-modulating bacteria for therapeutic purposes, which, when administered in an adequate amount, confer a health benefit on the host is being increasingly used. Majority of probiotic bacteria belong to the genera, Lactobacilli, Bifidobacterium, and Propionibacterium.

Probiotics have been documented to exert several actions on a wide variety of immune cells shifting it towards the anti-inflammatory pathway. ${ }^{1}$ Additionally, probiotic ac- tion could be augmented by using prebiotics such as inulin. This combination is known as symbiotic ${ }^{1}$ Aggour and colleagues (2021) ${ }^{1}$ reported on a randomized clinical trial that sought to explore the effectiveness of lozenges containing Lactobacillus acidophilus (L. acidophilus) and Bifidobacterium lactis, plus inulin in the treatment of minor RAS in adult and paediatric RAS patients.

\section{MATERIALS AND METHODS}

Sixty adult (group A) and 60 children patients (group B) with diagnosis of minor RAS were included in this randomized clinical trial $(R C T)$. Each group was divided into two subgroups as follows:

- Adult Group Al: ChocBalls (L. acidophilus containing lozenges)

- Adult Group All: (control) Oracure oral gel (15gm, Amun)

- Child Group BI: ChocBalls (L. acidophilus containing lozenges)

- Child Group BII: (control) Oracure oral gel (15gm, Amun)

The inclusion criteria for group A was as follows: (1) males and females aged 18-45 years old, (2) patients presenting with RAS with the following characteristics: 
(a) minor aphthous ulcers less than $48 \mathrm{~h}$ duration prior to enrolment, (b) size no greater than $10 \mathrm{~mm}$ in diameter, (c) a history that ulcers normally more than 5 days to resolve without treatment.

The inclusion criteria for group B are the same except age; children with RAS aged between 3 and 12 years were recruited for the study. The exclusion criteria are as follows: a known history of hypersensitivities, immunologic or systemic diseases, pregnancy, smoking, treatment with systemic steroid, or other immunomodulatory agents within 1 month before the study; use of nonsteroidal anti-inflammatory drugs or oral anti-histamines within 1 month prior to the study; treatment of the ulcer with any preparation or medication within $72 \mathrm{~h}$ prior to the study, and treatment with systemic anti-biotics within 2 weeks prior to the study and a history of adverse reactions to lactose or fermented milk products. Children with a positive family history of RAS were excluded. The nutritional status of the children was evaluated.

After taking a detailed history and clinical examination, all patients eligible for study participation were randomly assigned to the test subgroups ( $L$. acidophilus containing lozenges) or control subgroups. Adult lozenge was composed of the following: L. acidophilus 1.5 billion cfu, Bifidobacterium lactis 1.5 billion cfu and inulin $0.13 \mathrm{~g}$ per lozenge. For children, lozenge was composed of the following: L. acidophilus 0.5 billion cfu, Bifidobacterium lactis 0.5 billion cfu, and inulin $0.13 \mathrm{~g}$ per lozenge.

Patients were instructed to melt the $L$. acidophilus containing lozenges in the mouth slowly twice daily for 5 days (day 1 to day 5). The baseline parameters were taken and recorded on the day of the first visit. Effectiveness evaluations were made on the morning of day 3 visit and day 5 visit.

The index ulcer's size was measured on treatment days 0,3 , and 5 . To evaluate pain, a visual analog scale (VAS) consisting of a $10-\mathrm{cm}$ horizontal line between poles connoting no pain (origin) to unbearable pain was used. Subjects were told to mark the line with a vertical line at the point that best represented the present pain level of the ulcer.

The effectiveness indices (EI) of the ulcer size and pain improvement were measured at day 3 visit and day 5 visit. The effectiveness indices were evaluated on a 4-rank scale: (1) heal: El $\geq 95 \%$; (2) marked improvement: $\mathrm{El}<$ 95\%, but $\geq 70 \%$; (3) moderate improvement: $\mathrm{El}<70 \%$, but $\geq 30 \%$; and (4) no improvement: $\mathrm{El}<30 \%$. Participants were asked to estimate the average duration of episodes during the past 6 months and the potential to reduce the outbreak frequency of RAS within the next 6 months was investigated.

\section{RESULTS}

A total of 60 patients (39 females, 21 males) were included in group A (adult group) and 60 healthy children (34 females, 26 males) in group B (paediatric group). All patients enrolled in the study completed the trial. The baseline characteristics of the study groups showed no significant differences within each group.
For ulcer size reduction in adult patients (group A), the effectiveness index of $\mathrm{Al}$ was greater than All at both evaluation periods; however, the difference was statistically insignificant $(P=0.2149, P=0.08914$; respectively). Regarding pain reduction, subgroup Al had statistically greater effectiveness index when compared with subgroup All at both evaluation periods $(P=0.0455, P=0.0093$; respectively). For "improvement" rate, at day 3 , the subgroup $\mathrm{Al}$ had higher values for both ulcer size reduction and pain moderation (80\% vs. 53.33\%) when compared with subgroup All. At the day 5 visit, compared with subgroup All, subgroup Al maintained a greater improvement rate for pain moderation only. No change in outbreak frequency was reported within the 6 months next to treatment $(P>.05)$

For ulcer size reduction in paediatric patients (group B), the effectiveness index of $\mathrm{BI}$ is greater than $\mathrm{Bll}$ at both evaluation periods; however, the difference was statistically significant at only the day 5 visit $(P=0.0080)$. Regarding pain reduction, subgroup $\mathrm{Bl}$ had slightly greater effectiveness index when compared with subgroup BII at both evaluation periods; the difference was statistically insignificant $(P=0.1585, P=0.3787$; respectively). For "improvement" rate, at day 3 , the subgroup $\mathrm{BI}$ had higher values for both ulcer size reduction and pain moderation when compared with subgroup Bll $(90 \%$ vs. $73.33 \%$ and $63.33 \%$ vs. $53.33 \%$ respectively). At the day 5 visit, compared with subgroup BII, subgroup BI had a slightly higher improvement rate for ulcer size reduction and identical pain moderation. A statistically significant decrease in outbreak frequency was reported for subgroup $\mathrm{BI}$ within the 6 months next to treatment. The change was significantly different from subgroup Bll $(P<.05)$.

\section{CONCLUSIONS}

The researchers concluded that topical application of probiotics decreased pain intensity and accelerated RAS healing. The effectiveness in pain reduction was more evident in adult patients while acceleration of healing was greater in children.

\section{Implications for practice}

Probiotics is a well-tolerated, topical therapeutic agent for the management of minor RAS in both adults and children.

\section{Reference}

1. Aggour RL, Mahmoud SH, Abdelwhab A. Evaluation of the effect of probiotic lozenges in the treatment of recurrent aphthous stomatitis: a randomized, controlled clinical trial. Clinical Oral Investigations. 2021; 25: 2151-8. 